\title{
ClearF: a supervised feature scoring method to find biomarkers using class-wise embedding and reconstruction
}

\author{
Sehee Wang ${ }^{1}$, Hyun-Hwan Jeong ${ }^{2,3}$ and Kyung-Ah Sohn ${ }^{*}$ \\ From The 8th Annual Translational Bioinformatics Conference \\ Seoul, South Korea. 31 October - 2 November 2018
}

\begin{abstract}
Background: Feature selection or scoring methods for the detection of biomarkers are essential in bioinformatics. Various feature selection methods have been developed for the detection of biomarkers, and several studies have employed information-theoretic approaches. However, most of these methods generally require a long processing time. In addition, information-theoretic methods discretize continuous features, which is a drawback that can lead to the loss of information.
\end{abstract}

Results: In this paper, a novel supervised feature scoring method named ClearF is proposed. The proposed method is suitable for continuous-valued data, which is similar to the principle of feature selection using mutual information, with the added advantage of a reduced computation time. The proposed score calculation is motivated by the association between the reconstruction error and the information-theoretic measurement. Our method is based on class-wise low-dimensional embedding and the resulting reconstruction error. Given multi-class datasets such as a case-control study dataset, low-dimensional embedding is first applied to each class to obtain a compressed representation of the class, and also for the entire dataset. Reconstruction is then performed to calculate the error of each feature and the final score for each feature is defined in terms of the reconstruction errors. The correlation between the information theoretic measurement and the proposed method is demonstrated using a simulation. For performance validation, we compared the classification performance of the proposed method with those of various algorithms on benchmark datasets.

Conclusions: The proposed method showed higher accuracy and lower execution time than the other established methods. Moreover, an experiment was conducted on the TCGA breast cancer dataset, and it was confirmed that the genes with the highest scores were highly associated with subtypes of breast cancer.

Keywords: Feature selection, Feature scoring, Mutual information (MI), Breast cancer, Dimension reduction, Lowdimensional embedding, Reconstruction error, Principal component analysis (PCA)

\footnotetext{
* Correspondence: kasohn@ajou.ac.kr

${ }^{1}$ Department of Computer Engineering, Ajou University, Suwon 16499, South

Korea

Full list of author information is available at the end of the article
}

(c) The Author(s). 2019 Open Access This article is distributed under the terms of the Creative Commons Attribution 4.0 International License (http://creativecommons.org/licenses/by/4.0/), which permits unrestricted use, distribution, and reproduction in any medium, provided you give appropriate credit to the original author(s) and the source, provide a link to the Creative Commons license, and indicate if changes were made. The Creative Commons Public Domain Dedication waiver (http://creativecommons.org/publicdomain/zero/1.0/) applies to the data made available in this article, unless otherwise stated. 


\section{Background}

Feature selection or scoring techniques are essential for the solution of various problems in bioinformatics. Biomarkers are biological characteristics that can be used to predict the risks of diseases [1], and feature selection is a method used to detect them [2-4]. Various feature selection methods have been developed [5,6] and successfully used to identify biomarkers. The feature selection method is also used to reduce large-scale data. The data used in bioinformatics generally contains a relatively small number of samples compared to the number of features. Thus, the 'curse of dimensionality' [7] easily occurs, in which the number of required samples exponentially increases as the number of features increases. To overcome this drawback, a feature selection method is often applied to the selection of important features. It is therefore important to develop feature selection algorithms for the detection of biomarkers.

With respect to labels, feature selection can be divided into two categories: 1) supervised feature selection methods that utilize class label information and 2) unsupervised methods that do not use class labels [8]. Supervised feature selection methods are used to find useful biomarkers for the prediction of disease. There are several categories of supervised feature selection methods [8]. For example, statistical based methods use statistical measures to score each feature, and similarity based approaches select important features that can preserve data similarity.

Information-theoretic methods perform a feature selection using mutual information, which is a measure of the entropy and conditional entropy dependence between a variable of data and a label: I (X; Y), The mutual information between two random variables $\mathrm{X}$ (a random variable of data, feature) and $\mathrm{Y}$ (a random variable of label) can be expressed as follows:

$$
I(X ; Y)=H(X)-H(X \mid Y)
$$

where $H(X)$ is the entropy of the random variable $X$ and $\mathrm{H}(\mathrm{X} \mid \mathrm{Y})$ is the conditional entropy of the random variable of $\mathrm{X}$ given $\mathrm{Y}$. Information theoretic approaches are typically used to detect biomarkers [9-15]. However, in most cases, the processing time is long. In addition, information theoretic methods discretize continuous variables, which is a drawback that can lead to loss of information [16].

While feature selection reduces the dimension by selecting a subset of the overall features, low-dimensional embedding is a method that creates new low-dimensional feature representations without preserving the original features. Low-dimensional embedding is often used to obtain a low-dimensional representation by its application to problems that are difficult to process at higher dimensions. It is also used for noise removal through reconstruction [17].

Principal component analysis (PCA) is a typical low-dimensional embedding method that uses an orthogonal linear transformation for a high-dimensional data to a low-dimensional representation. It offers a high execution speed, and it is frequently used in many fields. However, it does not reflect nonlinearity. The kernel principal component analysis (KernelPCA) [18] is an improvement of the original PCA using the kernel method. Another low-dimensional embedding method is the autoencoder, which is a specific type of neural network. Recently, deep

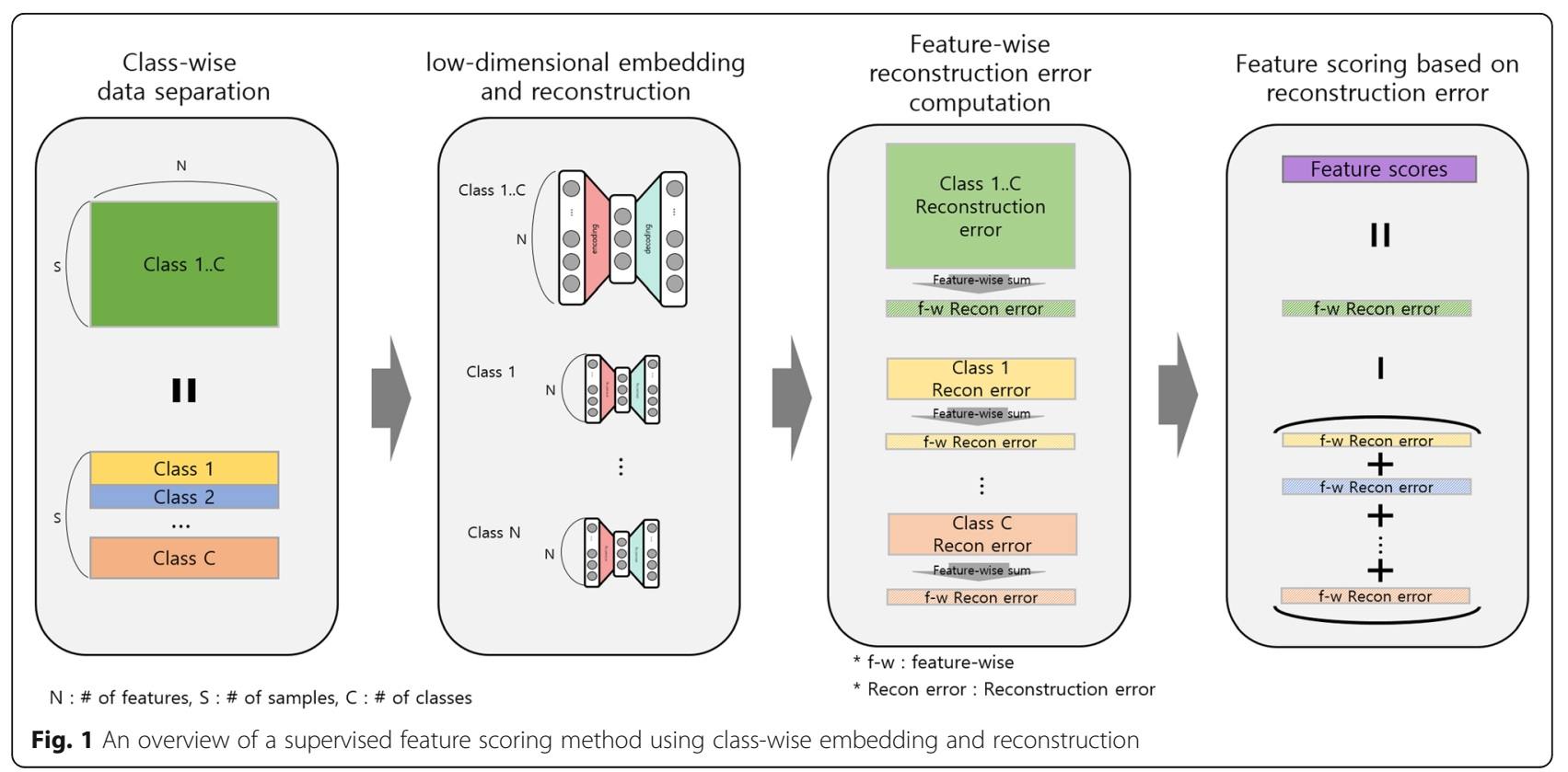




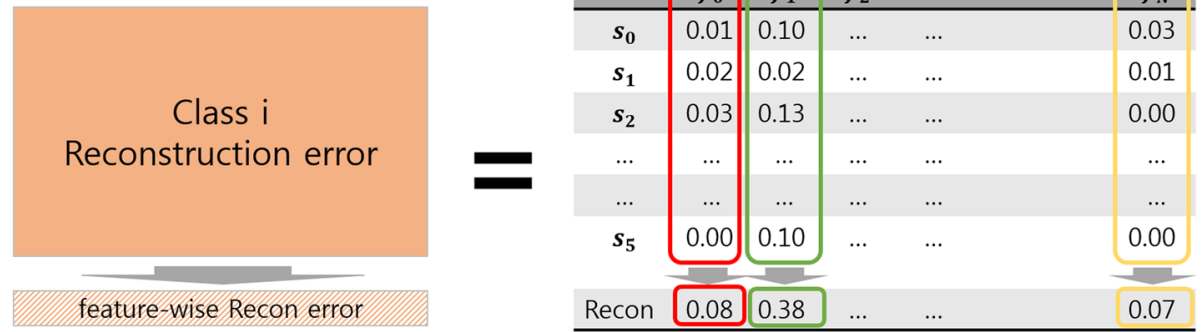

Fig. 2 An illustration of feature-wise reconstruction error computation

neural networks have been established, and the deep autoencoder has been widely used.

Low-dimensional embedding is effective in reducing data to low dimensions; however, it constructs a set of new features, and it is difficult to extract substantial interpretations of these features in the transformed space. It is therefore difficult to use it directly for biomarker detection in conjunction with low-dimensional embedding. Furthermore, given that most of the methods are unsupervised, it is difficult to utilize class label information.

In this paper, we propose a method for assigning supervised feature scores by applying unsupervised class-wise low-dimensional embedding. The performance of the proposed method is in accordance with the principle of feature selection based on mutual information. Moreover, the method addresses the problems we described above.

\section{Methods}

\section{Overview}

The proposed method is termed ClearF. It determines the feature score by calculating the reconstruction error after class-wise low-dimensional embedding, and it uses the property of the reconstruction error that

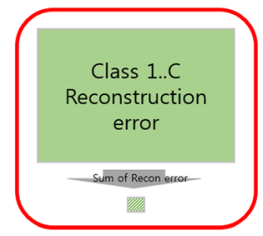

II

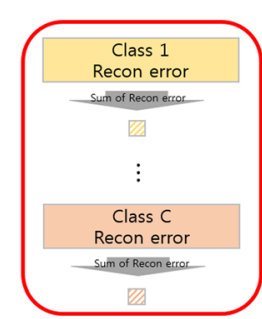

II

$$
\begin{gathered}
\operatorname{Score}(X)=\underbrace{\operatorname{Recon}_{\text {Class } 1 . . C}(X)}_{H(X ; Y)}-\sum_{i=1}^{C} \operatorname{Recon}_{\text {Class } i}(X) \\
H(X \mid Y)
\end{gathered}
$$

Fig. 3 Conversion of a mutual information concept to a formula using the reconstruction error differs by class. Figure 1 presents the overall structure of ClearF. First, class-wise division is performed on the entire dataset. In other words, if the number of class labels is $\mathrm{C}$, the entire dataset and data for each class are separated into $C+1$ datasets. Thereafter, the low-dimensional embedding and reconstruction are separately performed for each divided dataset. Any low-dimensional embedding method such as PCA, KernelPCA, and autoencoder can be applied to ClearF. The reconstruction error for each dataset is obtained by calculating the difference between reconstructed data and original data. The feature-specific reconstruction errors in each dataset are calculated by the feature-wise sum of reconstruction errors for each feature (Fig. 2). Finally, the feature-wise reconstruction error of all the data and the error of data for each class are used to derive the final feature score.

\section{Conversion of mutual information to reconstruction error- based concept}

Low-dimensional embedding reduces the size of high-dimensional data, and simplifies the data in the reduction process. Suppose we reduce a dataset to a very small dimension. If the characteristics of the data are complex, it is difficult to represent the characteristics of the

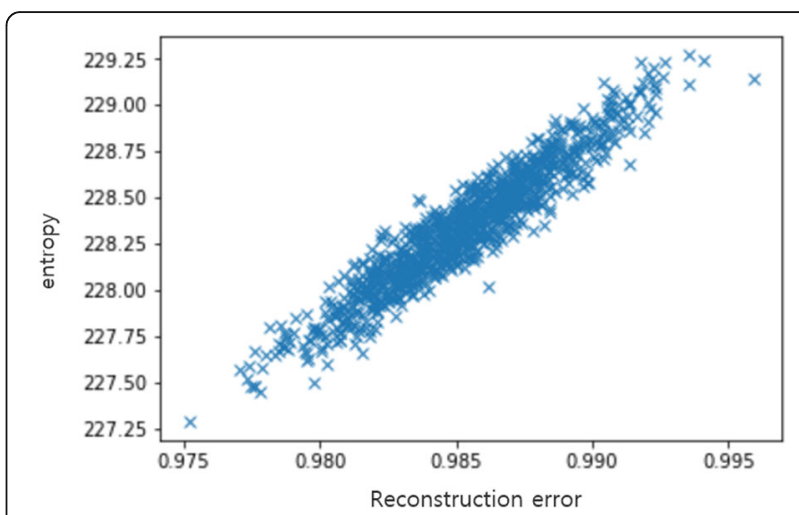

Fig. 4 Simulation results of the relationship between the entropy and reconstruction error 


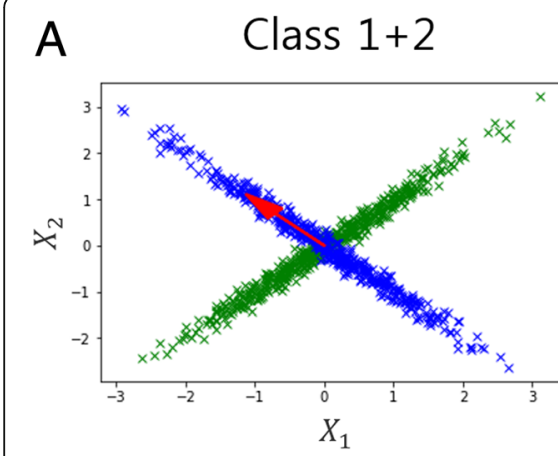

Reconstruction error

$X_{1}: 452.95, X_{2}: 465.62$
Class 1

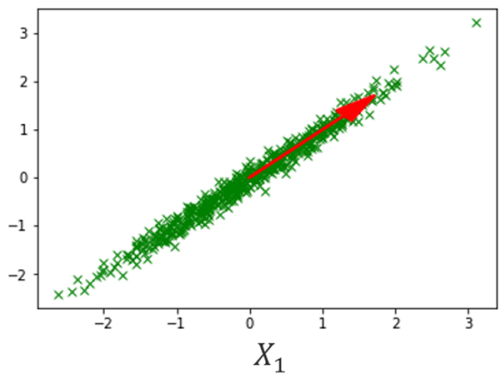

Reconstruction error

$X_{1}: 2.54, X_{2}: 2.56$
Class 2

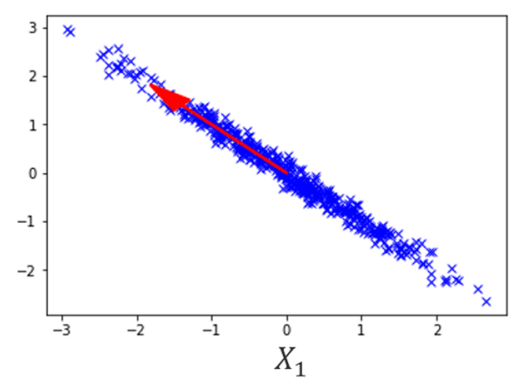

Reconstruction error $X_{1}: 2.47, X_{2}: 2.46$

Scores

$X_{1}: 447.94, X_{2}: 460.60$

B

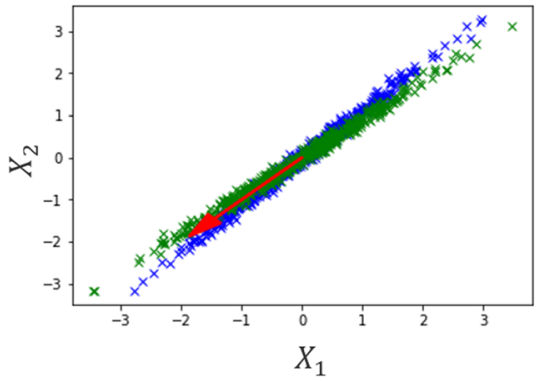

Reconstruction error $X_{1}: 5.28, X_{2}: 5.26$
Class 1

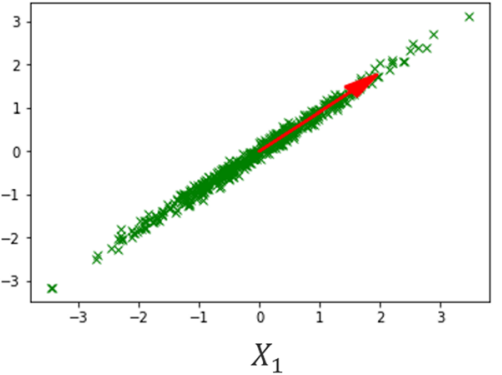

Reconstruction error

$X_{1}: 1.17, X_{2}: 1.44$
Class

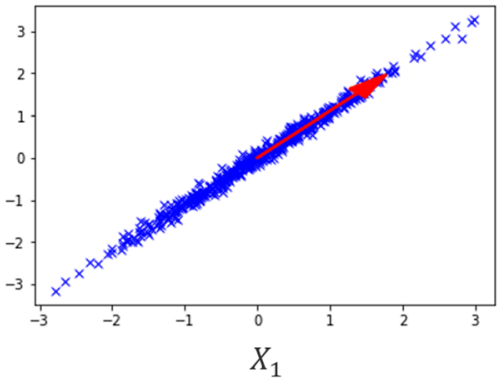

Reconstruction error $X_{1}: 1.40, X_{2}: 1.13$

$$
\begin{aligned}
& \text { Scores } \\
& X_{1}: 2.71, X_{2}: 2.69
\end{aligned}
$$

Fig. 5 Simulation results confirming the applicability of the scoring method for feature selection. The red arrows indicate the direction and size of the components. a shows the result on the dataset with two features that differ widely between classes, and $\mathbf{b}$ shows the result when there is little difference between classes

original data. Thus, information may be lost in the low-dimensional embedding process. On the other hand, if the characteristics of the data are comparatively simple, they can be sufficiently reflected in the low dimension. Intuitively, this leads to the hypothesis that the data is complex if the reconstruction error is high; otherwise the data is simple.
In information theory, entropy refers to the uncertainty of the data, which increases in accordance with an increase in the complexity of the data. This is similar to the characteristics of the reconstruction error described above. We use this to express entropy as a reconstruction error in a low-dimensional embedding process. Figure 3 presents the relationship between terms in the

Table 1 Detailed information of benchmark datasets

\begin{tabular}{llllll}
\hline Data set & Data type & Number of classes & Number of features & Number of samples & Data information \\
\hline Leukemia & Discrete & 2 & 7070 & 72 & SNP \\
ProstateGE & Continuous & 2 & 5966 & 102 & Gene expression \\
TOX171 & Continuous & 4 & 5748 & 171 & Gene expression \\
Lung & Continuous & 5 & 3312 & 203 & Gene expression \\
LungDiscrete & Discrete & 7 & 325 & 73 & SNP \\
\hline
\end{tabular}



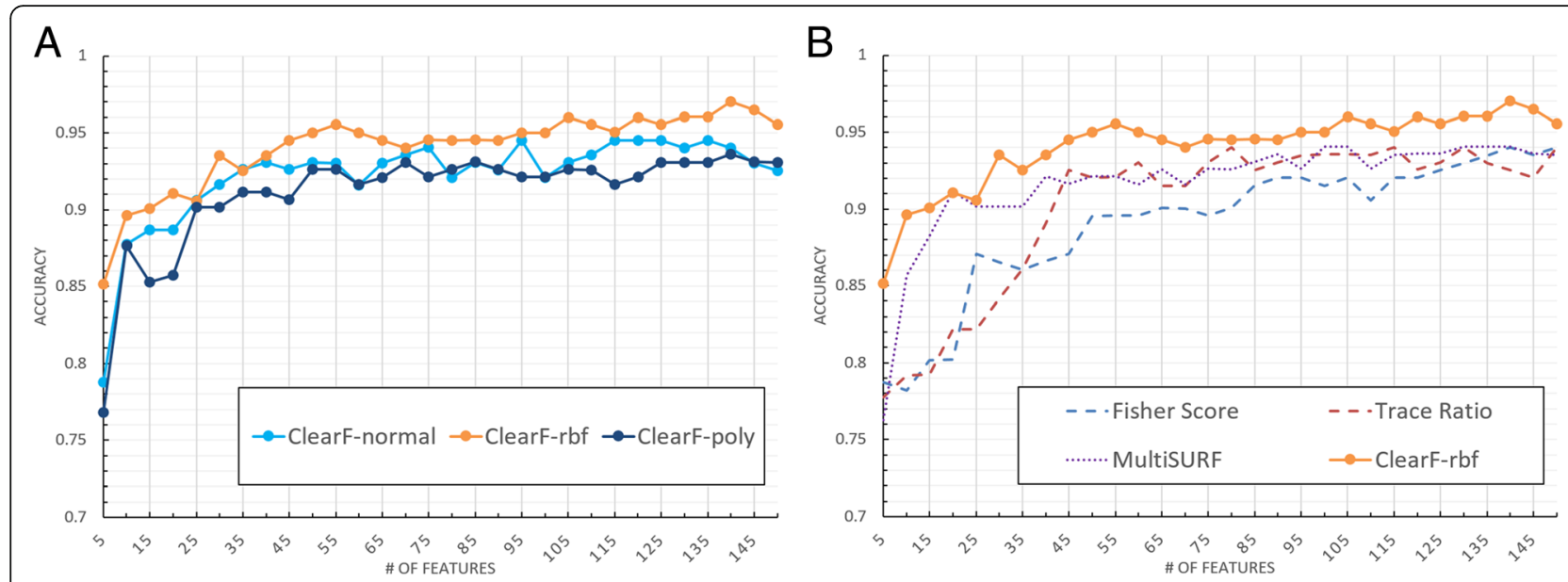

Fig. 6 Cross-validation accuracy for the Lung dataset with respect to the number of selected features. a presents the results of the PCA (ClearF-normal), KernelPCA with RBF kernel (ClearF-rbf) and KernelPCA with polynomial kernel (ClearF-poly) used in the proposed method; and $\mathbf{b}$ compares the results of the other algorithms with our method using the best result kernel

score $(\mathrm{X})$ and the entropy terms in the mutual information function. In particular, the entropy $\mathrm{H}(\mathrm{X})$ can be interpreted as the reconstruction error after the low-dimensional embedding of $X$. The conditional entropy $\mathrm{H}(\mathrm{X} \mid \mathrm{Y})$ corresponds to the reconstruction error when each existing label information is expressed. In Fig. 3, Recon Class $1 . . C_{C}(X)$ is a term of the reconstruction error after the low-dimensional embedding of data $X$; and $\sum_{i=0}^{C} \operatorname{Recon}_{\text {Class } i}(X)$ is the sum of the terms applied to each class separately. That is, the first part is calculated without label information and corresponds to $\mathrm{H}(\mathrm{X})$. The latter part is a reconstruction error in the state given the label information, and it corresponds to the conditional entropy under the label information.

\section{Reconstruction error-based feature scoring}

The reconstruction error of each feature in the feature-wise reconstruction error described in Fig. 2 is denoted as $\operatorname{Recon}_{\text {Class } i}\left(F_{j}\right)$. If it is associated with the score equation in Fig. 3, the following is obtained:

$$
\begin{gathered}
\operatorname{Score}(X)=\operatorname{Recon}_{\text {Class 1..C }}(X)-\sum_{1}^{C} \operatorname{Recon}_{i}(X) \\
=\sum_{1}^{F} \operatorname{Recon}_{1 . . C}\left(F_{j}\right)-\sum_{1}^{C} \sum_{1}^{F} \operatorname{Recon}_{i}\left(F_{j}\right) \\
=\sum_{1}^{F} \operatorname{Recon}_{1 . . C}\left(F_{j}\right)-\sum_{1}^{F} \sum_{1}^{C} \operatorname{Recon}_{i}\left(F_{j}\right)
\end{gathered}
$$
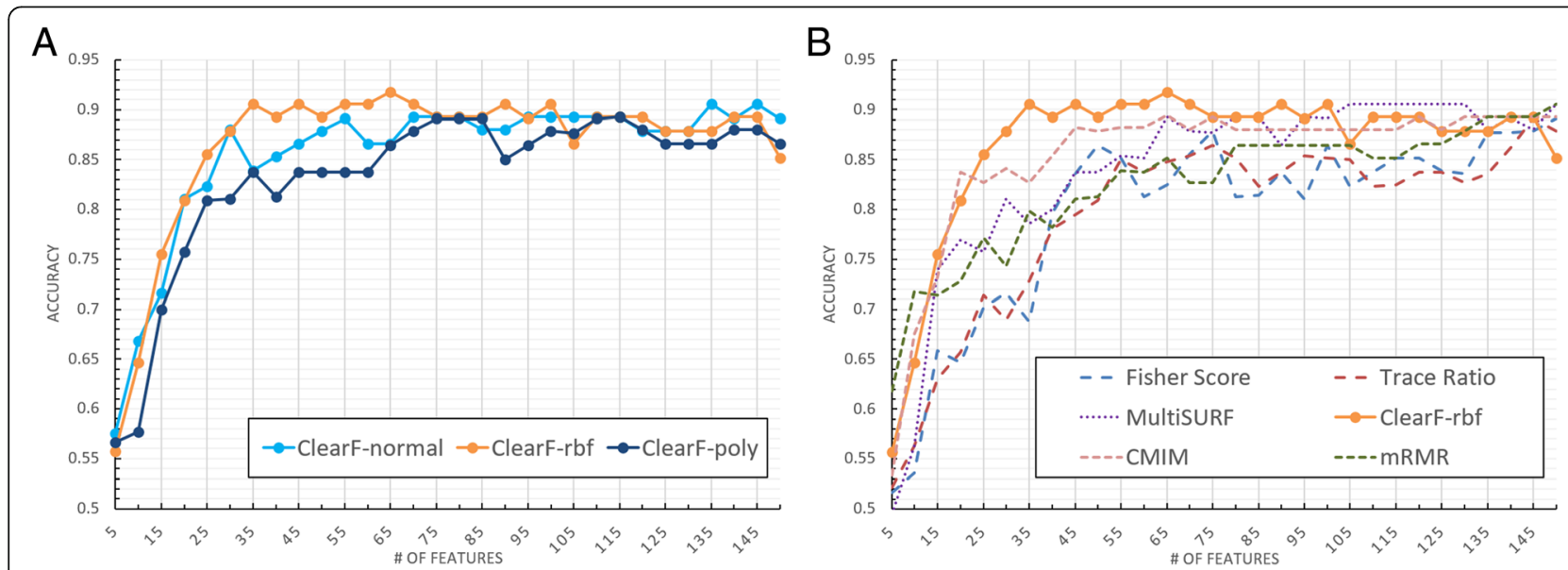

Fig. 7 Cross-validation accuracy for the LungDiscrete dataset with respect to the number of selected features. a presents the results of the PCA (ClearF-normal), KernelPCA with RBF kernel (ClearF-rbf) and KernelPCA with polynomial kernel (ClearF-poly) used in the proposed method; and $\mathbf{b}$ compares the results of the other algorithms with our method using the best result kernel 

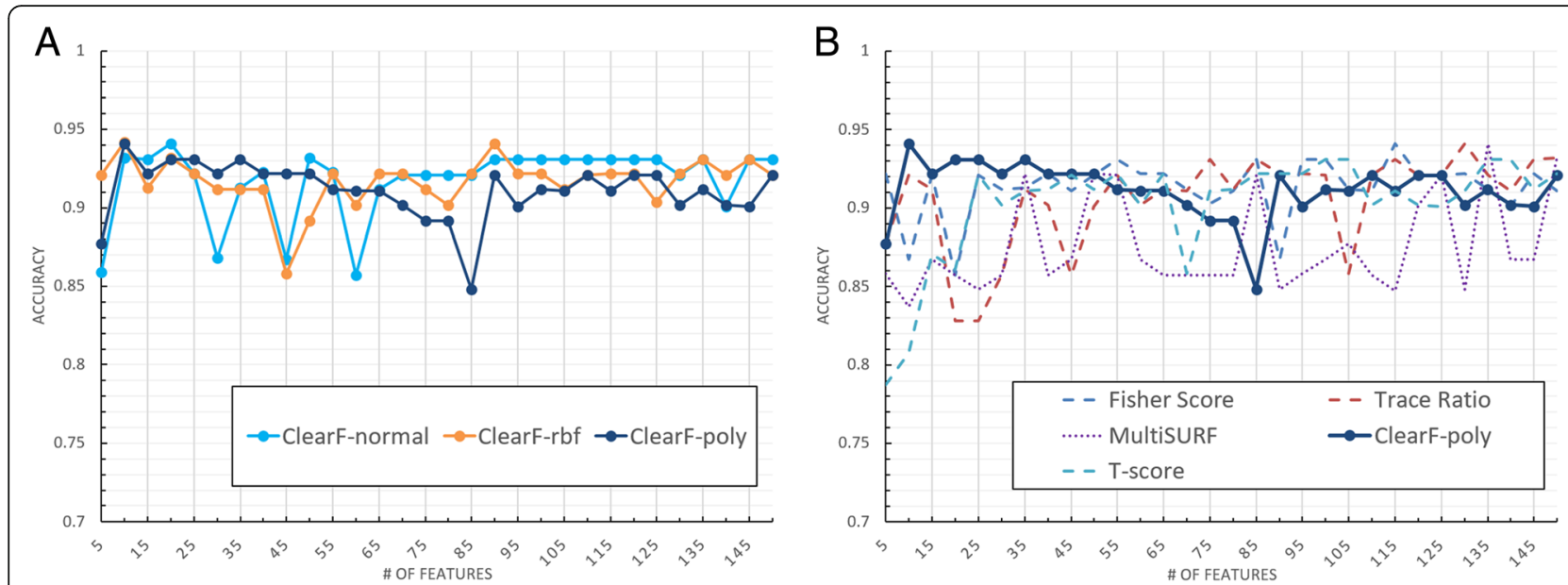

Fig. 8 Cross-validation accuracy for the ProstateGE dataset with respect to the number of features. a presents the results of the PCA (ClearFnormal), KernelPCA with RBF kernel (ClearF-rbf) and KernelPCA with polynomial kernel (ClearF-poly) used in the proposed method; and $\mathbf{b}$ compares the results of the other algorithms with our method using the best result kernel

The total score of $X$ is the sum of the scores calculated for each feature. The score calculated for each feature is the contribution of the feature to the total score; thus it can be called the score of the feature that can distinguish the label.

Our method can use any low-dimensional embedding method that is capable of reconstruction such as PCA, KernelPCA, and autoencoder. In the proposed method, the decreasing number of dimensions is a significant parameter. Since the purpose is to determine the reconstruction error difference of class-specific data with respect to that of the entire dataset, the same number of components or dimensions is used for the entire dataset and class-wise data.

\section{Results}

\section{Correlations between the entropy and reconstruction} error

To confirm the correspondence between the entropy and reconstruction error, a simulation was conducted.

The entropy of the multivariate Gaussian distribution can be calculated as follows, using the determinant of the covariance matrix [19]:

$$
H(X)=\frac{n}{2}+\frac{n}{2} \ln 2 \pi+\ln |\Sigma|
$$

where $n$ is the number of features in $\mathrm{X}$ and $\Sigma$ is the determinant of the covariance matrix. We used this to generate simulation data with a multivariate Gaussian distribution $N(0,1)$. Thereafter, the entropy was calculated to determine if there was a correlation with the reconstruction error.

The simulation data was generated to contain 100 features and 500 samples, and the entropy and reconstruction error of the generated data were calculated. Moreover, PCA was used for the low-dimensional embedding method, and the number of components in the PCA was set as one. We repeated this procedure 1000 times, and the results are presented in Fig. 4. The experimental results reveal that the entropy and the reconstruction error were highly correlated $\left(R^{2}=0.94\right)$.

\section{Simulation to verify the applicability of the proposed method}

We performed an experiment to confirm that our method works properly with a simulated dataset. The experiment was conducted to evaluate each score in the

Table 2 Average accuracy of using 5 to 50 features per method and dataset

\begin{tabular}{|c|c|c|c|c|c|c|c|c|c|}
\hline & Fisher score & Trace ratio & Multi SURF & ClearF normal & ClearF rbf & ClearF poly & CMIM & $\mathrm{mRMR}$ & t-score \\
\hline Leukemia & 0.945 & 0.945 & 0.945 & 0.973 & 0.959 & 0.945 & 0.973 & 0.959 & 0.959 \\
\hline ProstateGE & 0.912 & 0.857 & 0.857 & 0.868 & 0.912 & 0.922 & - & - & 0.902 \\
\hline TOX171 & 0.672 & 0.713 & 0.819 & 0.807 & 0.666 & 0.683 & - & - & - \\
\hline Lung & 0.865 & 0.841 & 0.901 & 0.916 & 0.935 & 0.902 & - & - & - \\
\hline LungDiscrete & 0.716 & 0.689 & 0.811 & 0.880 & 0.879 & 0.811 & 0.841 & 0.743 & - \\
\hline
\end{tabular}

The bold italic numbers indicate the best results for each dataset, and the bold non-italic numbers indicate the second-best result 

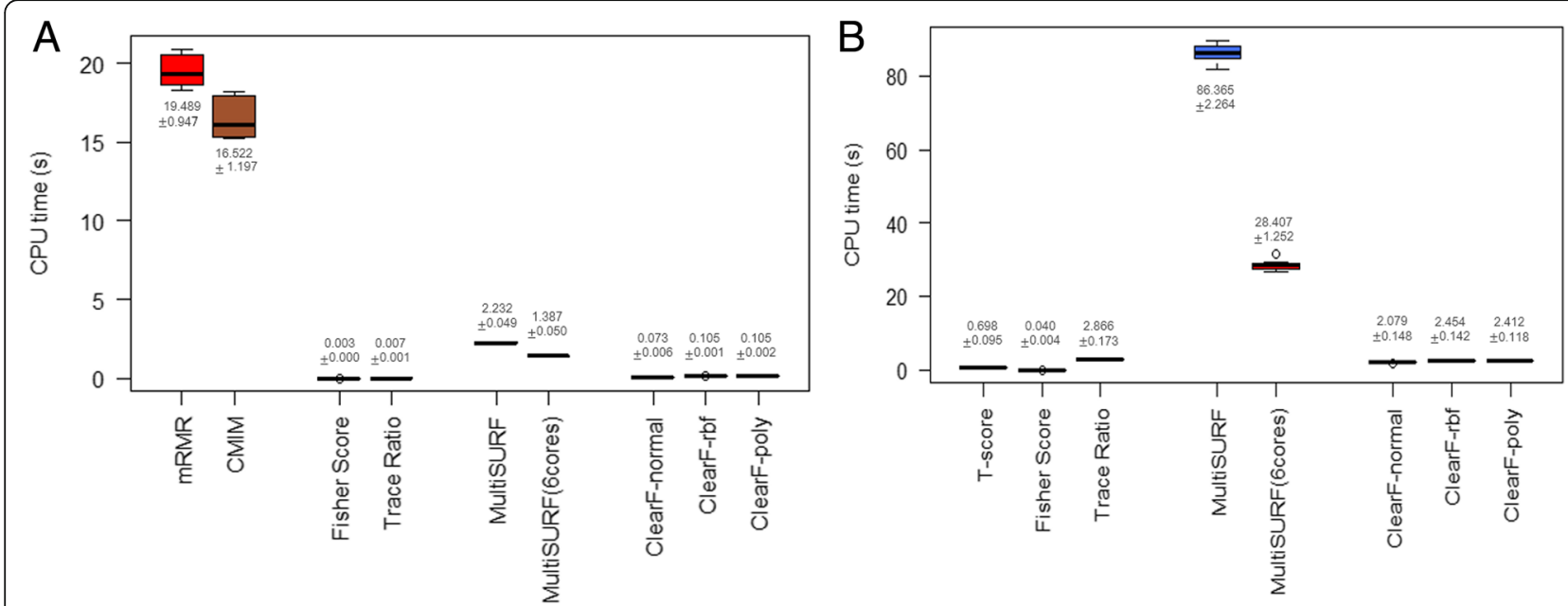

Fig. 9 Comparison of execution times for the LungDiscrete (a) and ProstatGE (b) dataset

following two cases: 1) when there is a large difference between the data for each class, and 2) when there is no difference between the data for each class. As shown in Fig. 5, we created two different datasets with two features and 500 samples. In particular, A was a dataset that contained two features that represented different trends by class, and B was a dataset that contained two features with fewer differences between classes. As in the previous experiments, PCA was used for the low-dimensional embedding, and the number of components in the PCA was set as one.

As shown in Fig. 5a, if combination of two features ( $X_{1}$ and $X_{2}$ in the figure) can easily differentiate data into the two classes, the reconstruction error for each class is very low. However, the reconstruction error of the entire dataset was very large; thus, the scores were 447.94 and 460.60, respectively, for each feature. On the other hand, in the case of Fig. 5b, the reconstruction error of the entire dataset was small; which resulted in low scores of 2.71 and 2.69, respectively. These results reveal that our scoring method produces high scores for the features that differ by class, and low when there is no difference by class.

\section{Performance validation for benchmark datasets}

To compare the performances of the proposed method and other established methods, five biological datasets were used from the benchmark datasets in [8]. Table 1 shows the details of the datasets. Several feature selection algorithms were used to conduct the benchmark tests. For performance comparison, we chose commonly used feature selection algorithms from each category described above. The selected algorithms were t-score [20] (binary class only), CMIM [21], mRMR [22] (discrete data only), Fisher score [23], Trace ratio [24],
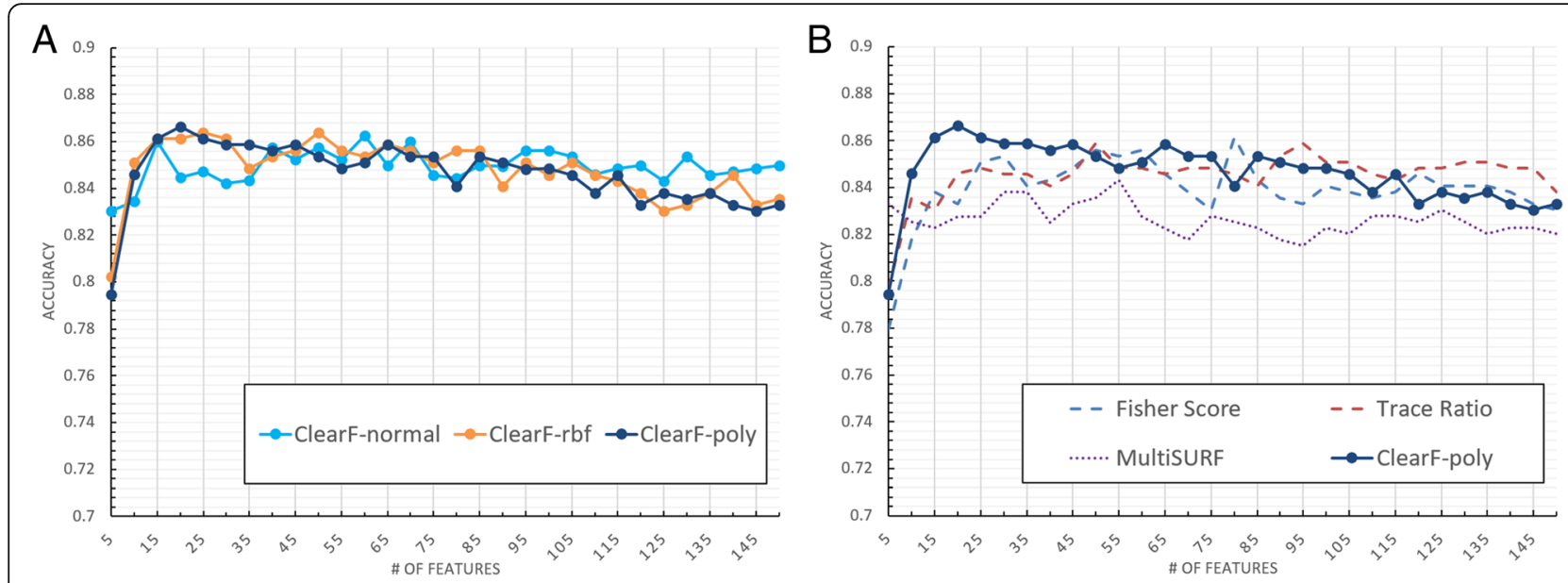

Fig. 10 Cross-validation accuracy for the TCGA dataset with respect to the number of features. a presents the results of the PCA (ClearF-normal), KernelPCA with RBF kernel (ClearF-rbf) and KernelPCA with polynomial kernel (ClearF-poly) used in the proposed method; and $\mathbf{b}$ compares the results of the other algorithms with our method using the best result kernel 
and multi-SURF [25]. For MultiSURF, we used the code provided by ReBATE [25], and the remaining algorithms were compared using code from scikit-features [8].

In order to confirm that the proposed method extracts more effective features for class label classification, the selected features from each method were used for classification, and the resulting accuracies were compared. We conducted a 10-fold cross validation in which the entire dataset was divided into 10 folds, one for test data and the other for training data. The feature selection algorithm was applied only to the training data to extract important features. The classification algorithm of support vector machine (SVM) with radial basis function (RBF) kernel was applied using only the selected features, and the average accuracy of the 10-fold cross validation was measured. The number of features to be selected in the above procedure was increased by five, and the process was the same as that used in the algorithm comparison in the previous study [8].

In our experimental setting, the low-dimensional embedding methods of PCA and KernelPCA were used. The kernels used for KernelPCA were the RBF kernel and the polynomial kernel with degree of three. Given that the component size is an important hyperparameter in the proposed method, we used a greedy search algorithm to find the optimal component size. The

Table 3 The top 30 genes with the highest scores obtained from the TCGA dataset

\begin{tabular}{|c|c|c|c|c|}
\hline Rank & Gene symbol & Entrez Gene Id & Gene Description & Score \\
\hline 1 & ERBB2 & 2064 & $\begin{array}{l}\text { v-erb-b2 erythroblastic leukemia viral oncogene } \\
\text { homolog 2, neuro/glioblastoma derived oncogene } \\
\text { homolog (avian) }\end{array}$ & 0.416 \\
\hline 2 & STARD3 & 10,948 & StAR-related lipid transfer (START) domain containing 3 & 0.339 \\
\hline 3 & PGAP3 & 93,210 & post-GPI attachment to proteins 3 & 0.295 \\
\hline 4 & FOXC1 & 2296 & forkhead box C1 & 0.276 \\
\hline 5 & CDKN2A & 1029 & $\begin{array}{l}\text { cyclin-dependent kinase inhibitor 2A (melanoma, } \\
\text { p16, inhibits CDK4) }\end{array}$ & 0.270 \\
\hline 6 & ORMDL3 & 94,103 & ORM1-like 3 (S. cerevisiae) & 0.259 \\
\hline 7 & GSDMB & 55,876 & gasdermin B & 0.245 \\
\hline 8 & B3GNT5 & 84,002 & UDP-GlcNAc:betaGal beta-1,3-N-acetylglucosaminyltransferase 5 & 0.236 \\
\hline 9 & PSMD3 & 5709 & proteasome (prosome, macropain) $26 \mathrm{~S}$ subunit, non-ATPase, 3 & 0.235 \\
\hline 10 & HAPLN3 & 145,864 & hyaluronan and proteoglycan link protein 3 & 0.231 \\
\hline 11 & CDCA7 & 83,879 & cell division cycle associated 7 & 0.222 \\
\hline 12 & PSAT1 & 29,968 & phosphoserine aminotransferase 1 & 0.216 \\
\hline 13 & C17orf37 & 84,299 & migration and invasion enhancer 1 & 0.215 \\
\hline 14 & GABRP & 2568 & gamma-aminobutyric acid (GABA) A receptor, pi & 0.215 \\
\hline 15 & TMSB15B & 286,527 & thymosin beta $15 \mathrm{~B}$ & 0.214 \\
\hline 16 & MED1 & 5469 & mediator complex subunit 1 & 0.208 \\
\hline 17 & CDCA2 & 157,313 & cell division cycle associated 2 & 0.207 \\
\hline 18 & FAM171A1 & 221,061 & family with sequence similarity 171 , member A1 & 0.203 \\
\hline 19 & CCNE1 & 898 & cyclin E1 & 0.197 \\
\hline 20 & CDK12 & 51,755 & cyclin-dependent kinase 12 & 0.194 \\
\hline 21 & DSC2 & 1824 & desmocollin 2 & 0.192 \\
\hline 22 & STAC & 6769 & $\mathrm{SH} 3$ and cysteine rich domain & 0.189 \\
\hline 23 & PADI2 & 11,240 & peptidyl arginine deiminase, type II & 0.189 \\
\hline 24 & RCOR2 & 283,248 & REST corepressor 2 & 0.179 \\
\hline 25 & IGF2BP2 & 10,644 & insulin-like growth factor 2 mRNA binding protein 2 & 0.176 \\
\hline 26 & $\mathrm{CDH} 3$ & 1001 & cadherin 3, type 1, P-cadherin (placental) & 0.175 \\
\hline 27 & ZNF695 & 57,116 & zinc finger protein 695 & 0.175 \\
\hline 28 & CLCN4 & 1183 & chloride channel 4 & 0.172 \\
\hline 29 & MEX3A & 92,312 & mex-3 homolog A (C. elegans) & 0.171 \\
\hline 30 & CBS & 875 & cystathionine-beta-synthase & 0.171 \\
\hline
\end{tabular}


component size was selected using only training data in each of 10 measurements of 10 -fold cross validation. First, the training data is further divided into three equal-sized subsets, two of which are used for model training and one of which is set as validation data. Let $C_{\min }$ be the number of samples of the smallest class in the dataset. We applied our method to training data for five different component sizes $\left(1, C_{\min } / 4, C_{\min } / 2,3\right.$. $C_{\text {min }} / 4$ and $C_{\text {min }}$ ). We selected the component size with the highest accuracy by applying SVM classifier. The mean squared error was calculated to measure the reconstruction error.

Figure 6 shows the result for the Lung dataset. We have performed the benchmarking using the previously mentioned algorithms, with the exception of T-score (only applicable to the binary class), mRMR, and CMIM (only applicable to discrete data), because the Lung dataset is a continuous and multi-class dataset. The proposed method demonstrated a relatively superior performance to those of the other algorithms. Especially, the RBF kernel showed a better performance than other methods regardless of the number of selected features.

The results for the LungDiscrete dataset are shown in Fig. 7. We excluded T-score because this dataset is a multi-class dataset. When the number of features was very small $(>20)$, mRMR and CMIM demonstrated comparable performances. However, when the number of features was larger than 30, ClearF-RBF archived a higher accuracy than other methods.

Figure 8 presents the results for the ProstateGE dataset. In the cases of using 10-30 features, the proposed method showed better performance than the other algorithms. The results of using more than 30 features were almost identical with the accuracy close to 0.9 .

The results for the Leukemia dataset are presented in Additional file 1: Figure S1. Most of all the methods demonstrated accuracies higher than 0.95 , and no significant difference between the performances of the methods were observed. In Additional file 1: Figure S2 presents the result of the Tox171 dataset. Although the results of the MultiSURF were relatively more accurate than those of the other methods, the results of our method using PCA were comparable.

For the purposes of feature selection, it is important that even a small number of selected features yield good results. The average accuracies for each method, from 5 to 50 features, are presented in Table 2. The bold italic numbers indicate the best results for each dataset, and

Table 4 Significant gene sets of overlap between MSigDB and Selected Genes

\begin{tabular}{|c|c|c|c|c|}
\hline Gene Set Name (\# Genes) & Description & $\begin{array}{l}\text { \# Genes in } \\
\text { Overlap }\end{array}$ & $p$-value & $\begin{array}{l}\text { FDR } \\
\text { q-value }\end{array}$ \\
\hline SMID_BREAST_CANCER_BASAL_UP (648) & $\begin{array}{l}\text { Genes up-regulated in basal subtype } \\
\text { of breast cancer samples. }\end{array}$ & 13 & $7.43 \mathrm{e}-17$ & 7.85 e-13 \\
\hline NIKOLSKY_BREAST_CANCER_17Q11_Q21_AMPLIPLICON (133) & $\begin{array}{l}\text { Genes within amplicon } 17 q 11-q 21 \\
\text { identified in a copy number alterations } \\
\text { study of } 191 \text { breast tumor samples. }\end{array}$ & 9 & 1.47 e-16 & 7.85 e-13 \\
\hline FARMER_BREAST_CANCER_CLUSTER_8 (7) & $\begin{array}{l}\text { Cluster 8: selected ERBB2 (GenelD = 2064) } \\
\text { amplicon genes clustered together } \\
\text { across breast cancer samples. }\end{array}$ & 5 & 1.75 e-15 & 6.23 e-12 \\
\hline VANTVEER_BREAST_CANCER_ESR1_DN (240) & $\begin{array}{l}\text { Down-regulated genes from the optimal } \\
\text { set of } 550 \text { markers discriminating breast } \\
\text { cancer samples by ESR1 (GenelD = 2099) } \\
\text { expression: ER(+) vs ER(-) tumors. }\end{array}$ & 9 & 3.23 e-14 & 8.16 e-11 \\
\hline SMID_BREAST_CANCER_LUMINAL_B_DN (564) & $\begin{array}{l}\text { Genes down-regulated in the luminal B } \\
\text { subtype of breast cancer. }\end{array}$ & 11 & $3.82 \mathrm{e}-14$ & 8.16 e-11 \\
\hline SMID_BREAST_CANCER_ERBB2_UP (147) & $\begin{array}{l}\text { Genes up-regulated in the erbb2 subype } \\
\text { of breast cancer samples, characterized } \\
\text { by higher expression of ERBB2 (GenelD }=2064) \text {. }\end{array}$ & 7 & 5.68 e-12 & $1.01 \mathrm{e}-8$ \\
\hline FARMER_BREAST_CANCER_BASAL_VS_LULMINAL (330) & $\begin{array}{l}\text { Genes which best discriminated between } \\
\text { two groups of breast cancer according to } \\
\text { the status of ESR1 and AR (GenelD = 2099;367): } \\
\text { basal (ESR1- AR-) and luminal (ESR1+ AR+). }\end{array}$ & 8 & 3.31 e-11 & 5.05 e-8 \\
\hline SMID_BREAST_CANCER_RELAPSE_IN_BONE_DN (315) & $\begin{array}{l}\text { Genes down-regulated in bone relapse of } \\
\text { breast cancer. }\end{array}$ & 7 & 1.18 e-9 & 1.58 e-6 \\
\hline DOANE_BREAST_CANCER_ESR1_DN (48) & $\begin{array}{l}\text { Genes down-regulated in breast cancer } \\
\text { samples positive for ESR1 (GenelD = 2099) } \\
\text { compared to the ESR1 negative tumors. }\end{array}$ & 4 & 2.81 e-8 & 3.34 e-5 \\
\hline FONTAINE_PAPILLARY_THYROID_CARCINOMA_UA_UP (66) & $\begin{array}{l}\text { Genes up-regulated in papillary thyroid } \\
\text { carcinoma (PTC) compared to other thyroid } \\
\text { tumors. }\end{array}$ & 4 & $1.03 \mathrm{e}-7$ & $1.1 \mathrm{e}-4$ \\
\hline
\end{tabular}


the bold non-italic numbers indicate the second-best result. The results show that the proposed method mostly showed a good performance. All the 10-fold cross validation accuracies and their standard deviations are shown in Additional file 1: Tables S1, S2, S3, S4, S5 and S6.

\section{Computational cost validation for benchmark datasets}

A comparison of the computational costs was performed to show that the proposed method has advantages of a reduced execution time. Among the benchmark datasets, LungDiscrete and ProstateGE were used, and the CPU time was measured by running each method 10 times for each data. In the case of MultiSURF, since it provides multi core implementation in ReBATE [25] code, separate experiments were conducted using a single core and six cores.

Figure 9a presents the results for the LungDiscrete dataset. It can be seen that the execution times of CMIM and mRMR, which are information-theoretic based methods, were much higher than those of the other methods. The results for the ProstateGE dataset are shown in Fig. 9b. The execution times of the other methods were lower than that of MultiSURF. The running time of our method is significantly shorter than those of the other feature selection methods except for the simple T-score or Fisher Score.

\section{Performance validation for the TCGA breast cancer dataset}

We performed an experiment using the TCGA (The Cancer Genome Atlas) gene expression data of patients with breast cancer. The genes with missing data were removed, and tested with 13,615 genes and 389 patients' data. Among the samples, 15 patients had HER2 positive, 280 had Luminal A, 37 had Luminal B, and 57 had basal-like subtypes.

Accuracy tests were performed on the TCGA breast cancer dataset in the same manner as the experiment in the benchmark dataset. The results are shown in Fig. 10, and they reveal that the proposed method yielded the best results for most of the sections.

Our method based on the KernelPCA (with polynomial kernel), which showed a good performance in the performance evaluation, was applied to all the data. The 30 genes with the highest score are presented in Table 3.

\section{Discussion}

Given the purpose of feature selection to identify important biomarkers, it is essential for a feature selection method to show good performance in selecting a small number of meaningful features. For the TCGA dataset as well as the benchmark datasets, the proposed method performed better than the other algorithms, especially in the results of selecting a small number of features $(10$ 50 ), which demonstrates the utility of our method for biomarker identification.

Among the highest-scoring genes detected using the proposed method, it is suspected that STARD3, PGAP3, ORMDL3, PSMD3 and HAPLN3 are the biomarkers of the HER2 + subtype [26-31], thus indicating that FOXC1 can identify basal-like subtypes in hereditary breast cancer cohorts [32]. In addition, the methylation status of CDKN2A exon2 has markedly higher methylation levels in luminal A and luminal B subtypes [33]. Moreover, gene expression may be a potential biomarker, as it is associated with methylation levels. B3GNT5 expression can also be a measure that distinguishes the

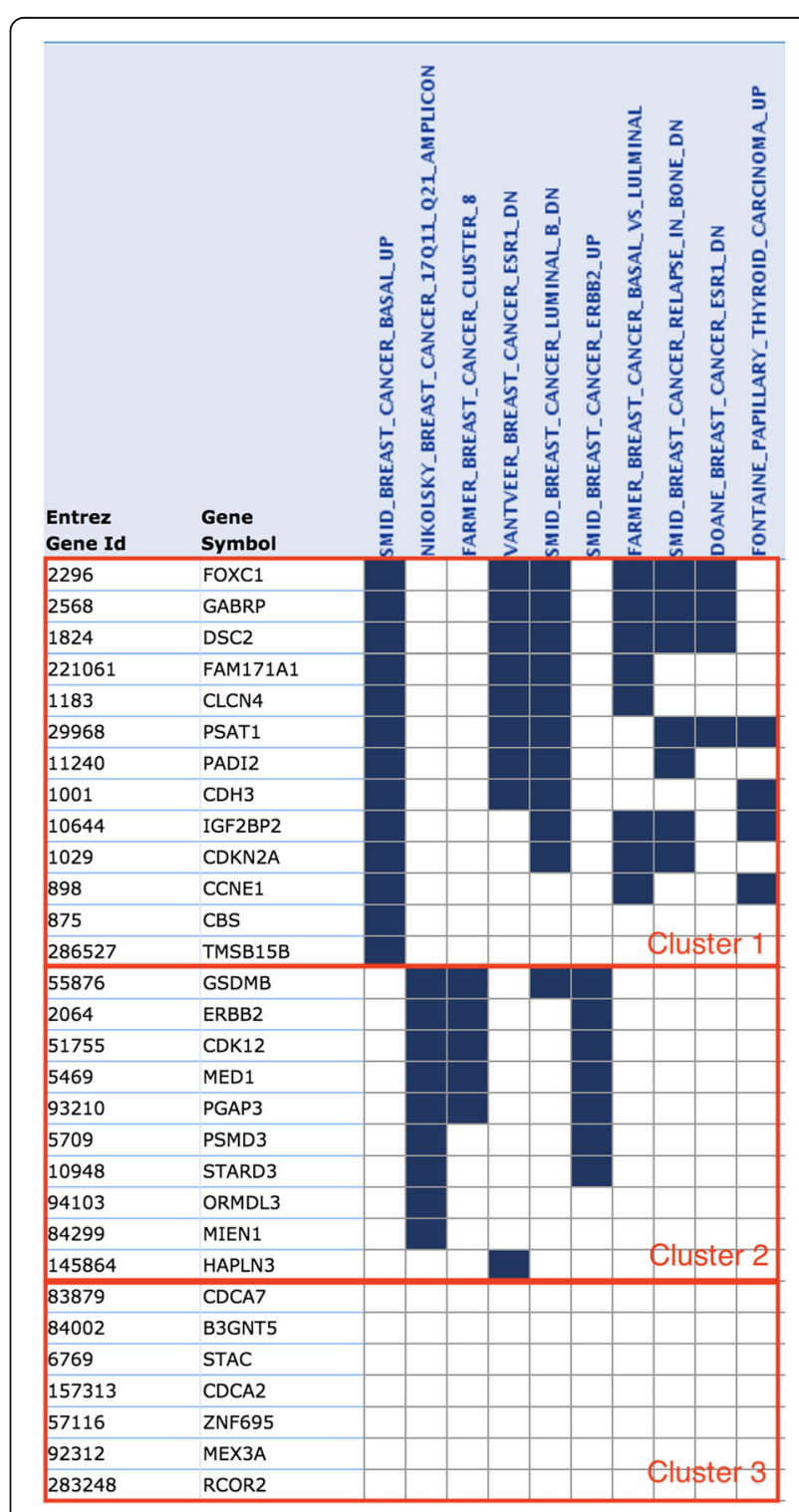

Fig. 11 Cluster information based on overlap of MsigDB and Selected Genes 
subtypes luminal A, B from basal-like as it is previously reported that the expression in basal-like samples is high, while the expression in luminal A and B is significantly lower [34].

Furthermore, GABRP, STAC, RCOR2, and IGFBP2 are the genes scored high by our method, but not by the other algorithms used in the performance comparison. The expression of GABA (A) receptor pi (GABRP) plays a role in initiation and progression of basal-like tumors, and has therapeutic potential in basal-like breast cancer [35]. In addition, the overexpression of IGFBP2 may be a feature of basal-like breast cancer that correlates with a low survival rate [36]. Moreover, RCOR2 replaces the need for Sox 2 expression in somatic cell reprogramming [37], whereas Sox2 has a positive expression in the basal-like subtype [38], although it has not been directly related to the breast cancer subtype. The similarity between somatic cell reprogramming and tumorigenesis [39] may suggest that Rcor2 is a potential biomarker.

We have also checked which of the top 30 genes belong to the $\mathrm{C} 2$ collection (curated gene sets) of MSigDB [40, 41]. The results are described in Table 4 and Fig. 11. Using the information in Table 4, genes can be classified into three clusters as shown in Fig. 11. As we have seen individually above, we find that Cluster 1 belongs to the up-regulated gene set in the basal-like subtype, and Cluster 2 is included in the Her2 + (triple negative) gene set.

Although the genes in Cluster 3 are not part of a significant gene set, CDCA2 and CDCA7 are genes involved in the cell division cycle-associated protein. Expression of several genes involved in cell division cycle-associated protein has been reported to cause shorter relapse free survival in patients with breast cancer [42]. Therefore, CDCA2 and CDCA7 may also be potential biomarkers. In addition, as previously described, Rcor2 is likely to be a potential biomarker, so other genes in Cluster 3 may also be potential biomarkers.

\section{Conclusion}

In this study, we developed a supervised feature selection algorithm that extracts useful features for the prediction of diseases or subtypes in biological data. By conducting simulation, we showed the applicability of the proposed method for feature selection. The experimental results revealed that our method has advantages both in terms of classification accuracy and execution speed, and is therefore useful in detecting biomarkers. This was also demonstrated by the extraction of meaningful genes and gene sets when applied to the TCGA dataset.

Additionally, we tried to use an auto-encoder for low-dimensional embedding, but the results were not stable. It is possible that the size of the dataset was not sufficiently big to show reasonable performance. In future work, the performance of an auto-encoder or other embedding methods should be evaluated using an appropriately sized datasets. In addition, we plan to conduct further studies on the selection of component numbers and their effects on the performance.

\section{Additional file}

\begin{abstract}
Additional file 1: Figure S1. Cross-validation accuracy for the Leukemia dataset with respect to the number of features. A presents the results of the PCA (ClearF-normal), KernelPCA with RBF kernel (ClearF-rbf) and KernelPCA with polynomial kernel (ClearF-poly) used in the proposed method; and B compares the results of the other algorithms with our method using the best result kernel. Figure S2. Cross-validation accuracy for the TOX171 dataset with respect to the number of features. A presents the results of the PCA (ClearF-normal), KernelPCA with RBF kernel (ClearF-rbf) and KernelPCA with polynomial kernel (ClearF-poly) used in the proposed method; and B compares the results of the other algorithms with our method using the best result kernel. Table S1. Detailed results of performance validation for Lung dataset. Table S2. Detailed results of performance validation for LungDiscrete dataset. Table S3. Detailed results of performance validation for ProstateGE dataset. Table S4. Detailed results of performance validation for Leukemia dataset. Table S5. Detailed results of performance validation for TOX171 dataset. Table S6.Detailed results of performance validation for TCGA dataset. (PDF 437 kb)
\end{abstract}

\section{Abbreviations}

MI: Mutual information; PCA: Principal component analysis; TCGA: The cancer genome atlas

\section{Acknowledgements}

We gratefully acknowledge the TCGA Consortium and all its members for the TCGA Project initiative, for providing tissues, sample, data processing and results available. Information about TCGA can be found at https://portal.gdc. cancer.gov/

\section{Funding}

This research was supported by the MSIT (Ministry of Science and ICT), Korea, under the ITRC (Information Technology Research Center) support program (IITP-2018-2018-0-01431) supervised by the IITP (Institute for Information \& communications Technology Promotion). Publication costs are funded by IITP (IITP-2018-2018-0-01431) and Ajou University.

Availability of data and materials

All data and material that are not presented in the main paper or additional supporting files are available from the corresponding author on request.

\section{About this supplement}

This article has been published as part of BMC Medical Genomics Volume 12 Supplement 5, 2019: Selected articles from the 8th Translational Bioinformatics Conference: Medical Genomics. The full contents of the supplement are available online at https://bmcmedgenomics.biomedcentral.com/articles/ supplements/volume-12-supplement-5.

\section{Authors' contributions}

SW, HJ, and KS designed and developed the study. SW implemented the idea and performed the experiments. SW, HJ, and KS performed the analysis. SW and HJ wrote the initial draft of the manuscript. KS supervised the whole analysis and writing. All authors have read and approved the final manuscript.

Ethics approval and consent to participate

Not applicable.

Consent for publication

Not applicable. 


\section{Competing interests}

The authors declare that they have no competing interests.

\section{Publisher's Note}

Springer Nature remains neutral with regard to jurisdictional claims in published maps and institutional affiliations.

\section{Author details}

'Department of Computer Engineering, Ajou University, Suwon 16499, South Korea. ${ }^{2}$ Jan and Dan Duncan Neurological Research Institute, Texas Children's Hospital, Houston, TX 77030, USA. ${ }^{3}$ Department of Molecular and Human Genetics, Baylor College of Medicine, Houston, TX 77030, USA.

Published: 11 July 2019

\section{References}

1. Group BDW, Atkinson AJ Jr, Colburn WA, De Gruttola VG, De Mets DL, Downing GJ, et al. Biomarkers and surrogate endpoints: preferred definitions and conceptual framework. Clin Pharmacol Ther. 2001;69:89-95.

2. He Z, Yu W. Stable feature selection for biomarker discovery. Comput Biol Chem. 2010;34:215-25. https://doi.org/10.1016/j.compbiolchem.2010.07.002.

3. Dessì N, Pascariello E, Pes B. A comparative analysis of biomarker selection techniques. Biomed Res Int. 2013;2013:1-10

4. Christin C, Hoefsloot HCJ, Smilde AK, Hoekman B, Suits F, Bischoff R, et al. A critical assessment of feature selection methods for biomarker discovery in clinical proteomics. Mol Cell Proteomics. 2013;12:263-76. https://doi.org/10. 1074/mcp.M112.022566.

5. Lee I-H, Lushington GH, Visvanathan M. A filter-based feature selection approach for identifying potential biomarkers for lung cancer. J Clin Bioinforma. 2011;1:11. https://doi.org/10.1186/2043-9113-1-11.

6. Abeel T, Helleputte T, Van de Peer Y, Dupont P, Saeys Y. Robust biomarker identification for cancer diagnosis with ensemble feature selection methods. Bioinformatics. 2009:26:392-8.

7. Bengio Y, Courville A, Vincent P. Representation learning: a review and new perspectives. IEEE Trans Pattern Anal Mach Intell. 2013;35:1798-828.

8. Li J, Cheng K, Wang S, Morstatter F, Trevino RP, Tang J, et al. Feature Selection: A Data Perspective. 2016; January. doi:https://doi.org/10.1145/3136625.

9. Parmar C, Grossmann P, Bussink J, Lambin P, Aerts HJWL. Machine Learning methods for Quantitative Radiomic Biomarkers. Sci Rep. 2015;5:1-11. https:// doi.org/10.1038/srep13087.

10. Chanda P, Sucheston L, Zhang A, Ramanathan M. The interaction index, a novel information-theoretic metric for prioritizing interacting genetic variations and environmental factors. Eur J Hum Genet. 2009;17:1274-86. https://doi.org/10.1038/ejhg.2009.38.

11. Chanda P, Sucheston L, Liu S, Zhang A, Ramanathan M. Information-theoretic gene-gene and gene-environment interaction analysis of quantitative traits. BMC Genomics. 2009;10:509.

12. Jeong $\mathrm{H}$, Sohn K-A. Relevance epistasis network of gastritis for intrachromosomes in the Korea associated resource (KARE) cohort study Genomics Inform. 2014;12:216. https://doi.org/10.5808/GI.2014.12.4.216

13. Leem S, Jeong HH, Lee J, Wee K, Sohn KA. Fast detection of high-order epistatic interactions in genome-wide association studies using information theoretic measure. Comput Biol Chem. 2014;50:19-28. https://doi.org/10. 1016/j.compbiolchem.2014.01.005

14. Jeong HH, Leem S, Wee K, Sohn KA. Integrative network analysis for survivalassociated gene-gene interactions across multiple genomic profiles in ovarian cancer. J Ovarian Res. 2015;8:1-15. https://doi.org/10.1186/s13048-015-0171-1.

15. Wang S, Jeong H, Kim D, Wee K, Park H-S, Kim S-H, et al. Integrative information theoretic network analysis for genome-wide association study of aspirin exacerbated respiratory disease in Korean population. BMC Med Genet. 2017;10:31. https://doi.org/10.1186/s12920-017-0266-1.

16. Dyhr-Nielsen M. Loss of information by discretizing hydrologic series. Hydrol Pap. 1972; October;54:65.

17. Hira ZM, Gillies DF. A review of feature selection and feature extraction methods applied on microarray data. Adv Bioinforma. 2015;2015. https://doi. org/10.1155/2015/198363.

18. Scholkopf B, Smola a J, Muller KR. Kernel principal component analysis. Comput Vis Math Methods Med Biomed Image Anal. 2012;1327:583-8. https://doi.org/10.1162/089976698300017467.

19. Ahmed NA, Gokhale DV. Entropy expressions and their estimators for multivariate distributions. IEEE Trans Inf Theory. 1989;35:688-92.
20. Davis JC. Statistics and data analysis in geology. 2nd ed. New York: Wiley; 1990

21. Fleuret $F$. Fast binary feature selection with conditional mutual information. J Mach Learn Res. 2004;5:1531-55.

22. Peng H, Long F, Ding C. Feature selection based on mutual information criteria of max-dependency, max-relevance, and min-redundancy. IEEE Trans Pattern Anal Mach Intell. 2005;27:1226-38.

23. Duda RO, Hart PE, Stork DG. Pattern classification. New york: Wiley; 2012.

24. Nie F, Xiang S, Jia Y, Zhang C, Yan S. Trace Ratio Criterion for Feature Selection Twenty-Third AAAI Conf Artif Intell; 2008. p. 671-6.

25. Urbanowicz RJ, Olson RS, Schmitt P, Meeker M, Moore JH. Benchmarking relief-based feature selection methods for bioinformatics data mining; 2017. p. 1-52. https://doi.org/10.1016/j.jbi.2018.07.015.

26. Yersal O. Biological subtypes of breast cancer: prognostic and therapeutic implications. World J Clin Oncol. 2014;5:412. https://doi.org/10.5306/wjco.v5.33.412.

27. Sircoulomb F, Bekhouche I, Finetti P, Adélaïde J, Hamida AB, Bonansea J, et al. Genome profiling of ERBB2-amplified breast cancers. BMC Cancer. 2010; 10:539. https://doi.org/10.1186/1471-2407-10-539.

28. Kauraniemi P, Kallioniemi A. Activation of multiple cancer-associated genes at the ERBB2 amplicon in breast cancer. Endocr Relat Cancer. 2006;13:39-49.

29. Li Y, Tang XQ, Bai Z, Dai X. Exploring the intrinsic differences among breast tumor subtypes defined using immunohistochemistry markers based on the decision tree. Sci Rep. 2016:6(June):1-13. https://doi.org/10.1038/srep35773.

30. Loussouarn D, Campion L, Leclair F, Campone M, Charbonnel C, Ricolleau G, et al. Validation of UBE2C protein as a prognostic marker in node-positive breast cancer. Br J Cancer. 2009;101:166-73.

31. Kuo S-J, Chien S-Y, Lin C, Chan S-E, Tsai H-T, Chen D-R. Significant elevation of CLDN16 and HAPLN3 gene expression in human breast cancer. Oncol Rep. 2010;24:759-66.

32. Johnson J, Choi M, Dadmanesh F, Han B, Qu Y. FOXC1 identifies basal-like breast cancer in a hereditary breast cancer cohort. Oncotarget. 2016;7(46): $75729-38$

33. Spitzwieser M, Entfellner E, Werner B, Pulverer W, Pfeiler G, Hacker S, et al, Hypermethylation of CDKN2A exon 2 in tumor, tumor-adjacent and tumordistant tissues from breast cancer patients. BMC Cancer. 2017;17:1-16.

34. Potapenko IO, Lüders T, Russnes HG, Helland Å, Sørlie T, Kristensen VN, et al. Glycan-related gene expression signatures in breast cancer subtypes; relation to survival. Mol Oncol. 2015:9:861-76.

35. Sizemore GM, Sizemore ST, Seachrist DD, Keri RA. GABA(a) receptor pi (GABRP) stimulates basal-like breast cancer cell migration through activation of extracellular-regulated kinase 1/2 (ERK1/2). J Biol Chem. 2014;289:24102-13.

36. Barghash A, Helms V, Kessler SM. Overexpression of IGF2 mRNA-binding protein 2 (IMP2/p62) as a feature of basal-like breast Cancer correlates with short survival. Scand J Immunol. 2015;82:142-3.

37. Yang $P$, Wang $Y$, Chen J, Li H, Kang L, Zhang Y, et al. RCOR2 is a subunit of the LSD1 complex that regulates ESC property and substitutes for SOX2 in reprogramming somatic cells to pluripotency. Stem Cells. 2011;29:791-801.

38. Lengerke C, Fehm T, Kurth R, Neubauer H, Scheble V, Müller F, et al. Expression of the embryonic stem cell marker SOX2 in early-stage breast carcinoma. BMC Cancer. 2011;11:42.

39. Friedmann-Morvinski D, Verma IM. Dedifferentiation and reprogramming: origins of cancer stem cells. EMBO Rep. 2014;15:244-53.

40. Subramanian A, Tamayo P, Mootha VK, Mukherjee S, Ebert BL, Gillette MA, et al. Gene set enrichment analysis: a knowledge-based approach for interpreting genome-wide expression profiles. Proc Natl Acad Sci. 2005;102: 15545-50. https://doi.org/10.1073/pnas.0506580102.

41. Liberzon A, Subramanian A, Pinchback R, Thorvaldsdóttir H, Tamayo P, Mesirov JP. Molecular signatures database (MSigDB) 3.0. Bioinformatics. 2011;27:1739-40.

42. Phan NN, Wang C, Li K, Chen C. Distinct expression of CDCA3, CDCA5 and CDCA8 leads to shorter relapse free survival in breast cancer patient. Oncotarget. 2018:9:6977-92. 